Article

\title{
Driving Waveform Design with Rising Gradient and Sawtooth Wave of Electrowetting Displays for Ultra-Low Power Consumption
}

\author{
Wei Li ${ }^{1,2}$, Li Wang 2,3,*, Taiyuan Zhang ${ }^{1,2}$, Shufa Lai ${ }^{1,2}$, Linwei Liu ${ }^{1,2}$, Wenyao He ${ }^{1,2}$, \\ Guofu Zhou ${ }^{1,2,3}$ and Zichuan Yi ${ }^{2,4}$ (D) \\ 1 Guangdong Provincial Key Laboratory of Optical Information Materials and Technology \& Institute of Electronic \\ Paper Displays, South China Academy of Advanced Optoelectronics, South China Normal University, \\ Guangzhou 510006, China; wei.li@guohua-oet.com (W.L.); taiyuan.zhang@guohua-oet.com (T.Z.); \\ shufa.lai@guohua-oet.com (S.L.); linwei.liu@guohua-oet.com (L.L.); wenyao.he@guohua-net.com (W.H.); \\ guofu.zhou@m.scnu.edu.cn (G.Z.) \\ 2 Shenzhen Guohua Optoelectronics Tech. Co., Ltd., Shenzhen 518110, China; yizichuan@163.com \\ 3 Academy of Shenzhen Guohua Optoelectronics, Shenzhen 518110, China \\ 4 College of Electron and Information, University of Electronic Science and Technology of China, \\ Zhongshan Institute, Zhongshan 528402, China \\ * Correspondence: wang.li@guohua-oet.com or creekxi@163.com; Tel.: +86-0755-29415855
}

Received: 26 November 2019; Accepted: 20 January 2020; Published: 28 January 2020

check for updates

\begin{abstract}
As a kind of paper-like display technology, power consumption is a very important index for electrowetting displays (EWDs). In this paper, the influence of driving waveforms on power consumption of the EWDs is analyzed, and a driving waveform with rising gradient and sawtooth wave is designed to reduce the power consumption. There are three stages in the proposed driving waveform. In the initial stage, the driving voltage is raised linearly from the threshold to the maximum value to reduce the invalid power consumption. At the same time, the oil breakup can be prohibited. And then, a section of sawtooth wave is added for suppressing oil backflow. Finally, there is a section of resetting wave to eliminate the influence of charge leakage. Experimental results show that the power consumption of the ultra-low power driving waveform is $1.85 \mathrm{~mW}$, which is about $38.13 \%$ lower than that of the conventional used square wave $(2.99 \mathrm{~mW})$, when the aperture ratio is $65 \%$.
\end{abstract}

Keywords: driving waveform; power consumption; electrowetting display; aperture ratio; response speed

\section{Introduction}

The electrowetting display (EWD) is a kind of reflective display technology, which has the advantages of video updating rate, high contrast ratio and low power consumption. Its working mechanism is to manipulate the interfacial tension of the polar liquid in a pixel by applying electric field, to achieve the control of optical switch and gray scale display [1]. The EWD technology has been proposed by G. Beni in 1981 at the first time [2]. In 2003, Robert A. Hayes realized high quality EWD [1], and then, a three-color EWD with vertical stack structure was proposed in 2010 [3].

In order to improve the display quality of EWDs, interface materials, pixel structures, fluidic motion mechanism and the relationship between electro-optical performances and applied voltage have been studied [4-7]. At the same time, the influence of driving waveforms on the performance of EWDs have been also studied. A decoupling driving waveform has been proposed to diminish the induced voltage stress [8]. However, the response time of the EWD is as long as $30 \mathrm{~ms}$ which cannot be used to display video. Then, a driving waveform was proposed for realizing the display of multi gray 
scales according to the characteristics of EWDs, square wave with high frequency is used for reducing flicker, a reset process is added to eliminate the influence of charge leakage [9]. However, the high frequency will lead to high power consumption. At the same time, a method was proposed to reduce oil breakup by adding a voltage rising gradient in the driving waveform [10]. However, the rising gradient can increase the response time of EWDs. Recently, a dynamic and asymmetric driving waveform was proposed to achieve more gray levels with limited voltage levels [11]. Although the power consumption has been decreased by restricting the maximum voltage of the dynamic and asymmetric driving waveform, the maximum aperture ratio cannot be achieved due to the limitation of the maximum voltage.

In this paper, according to EWD's equivalent circuit model, the power consumption of conventional driving waveforms is calculated and measured. Then, the relationship between the driving waveform and the power consumption is studied based on the experimental data. A driving waveform for ultra-low power consumption is proposed by reducing the driving voltage and shortening the effective driving time in each frame. With the same display effect, the proposed driving waveform can achieve lower power consumption than conventional waveforms.

\section{Principle of EWDs}

Gray scale is realized in EWDs by applying an external voltage to control the movement of colored oil. Its essence is an optical switch, which has excellent gray scale display characteristics [12]. As shown in Figure 1a, the colored oil in the pixel spreads naturally and covers the whole pixel when no external voltage is applied, and the color of the oil is displayed; the colored oil is pushed to a corner in the pixel when the external voltage is applied, and the color of the substrate is displayed, as shown in Figure $1 \mathrm{~b}$. Figure $1 \mathrm{c}$ is the top view of oil spreading. Figure $1 \mathrm{~d}$ is the top view of oil contracting. The different degrees of oil contraction represent different optical states, which are characterized by the aperture ratio. The aperture ratio is the proportion of the opening area in the whole pixel. The formula is defined as follows [5]:

$$
W_{A}(V)=\left(1-\frac{S_{\text {oil }}(V)}{S_{\text {pix }}}\right) \times 100 \%
$$

In Equation (1), $W_{A}(V)$ represents the aperture ratio, $S_{\text {oil }}(V)$ and $S_{\text {pix }}$ represent the surface area of oil in a single pixel and the surface area of the whole pixel respectively, $V$ represents the voltage applied to the EWD, and the area of pixel wall is ignored in calculating the aperture ratio. The pixel wall is a transparent grid structure which can divide the EWD into several pixels.

The pixel structure of the EWD is shown in Figure 2a, which is mainly composed of glass substrate, ITO (Indium Tin Oxides) guide electrode, hydrophobic insulation layer, pixel wall, color oil, and polar liquid [13]. The three-dimensional structure of an EWD pixel is shown in Figure $2 b$.

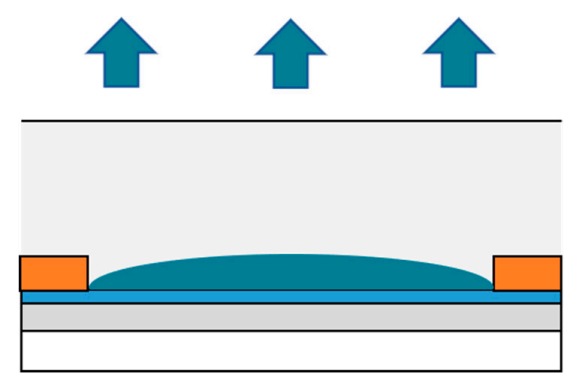

(a)

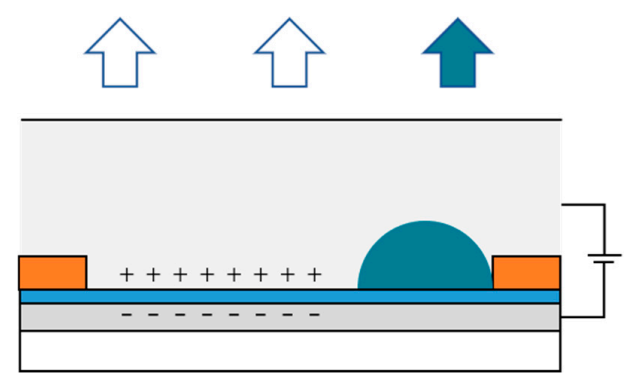

(b)

Figure 1. Cont. 


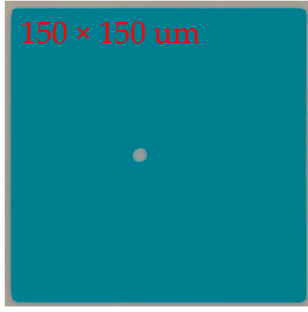

(c)

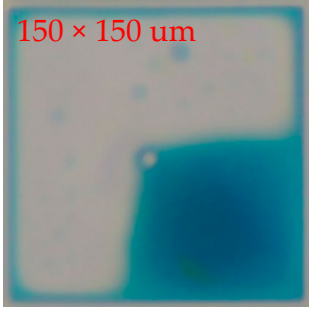

(d)

Figure 1. Principle of the electrowetting display (EWD). (a) Without applied voltage, the color of oil is displayed in a pixel. (b) With applied voltage, the color of substrate is displayed. (c) The top view when the pixel is turned off. (d) The top view when the pixel is turned on.

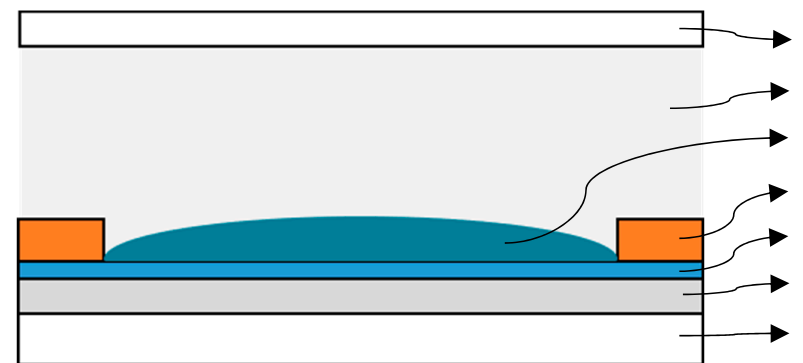

Top plate

$\mathrm{NaCl}$ solution

Colored oil

Pixel wall

Hydrophobic insulator

ITO

White substrate

(a)

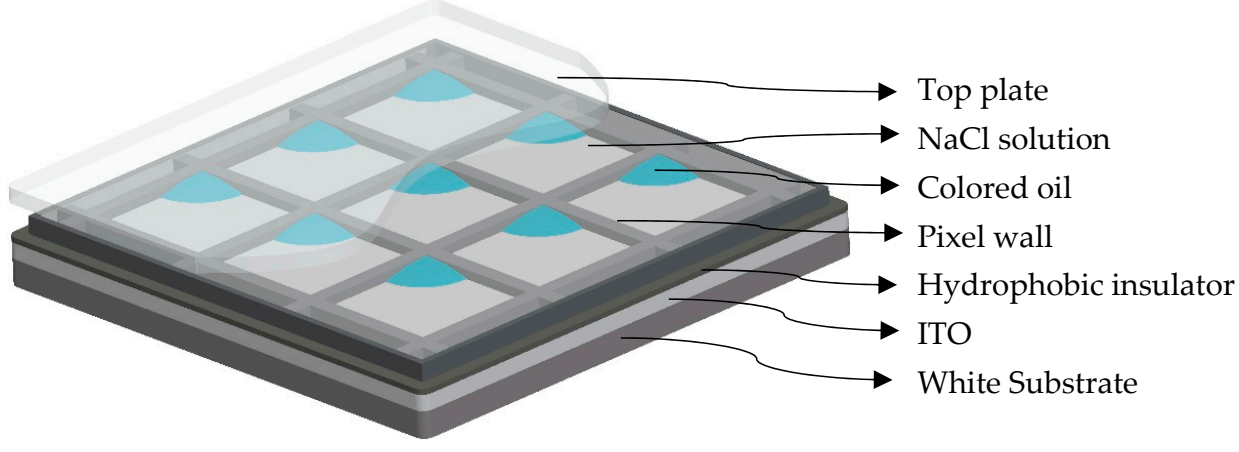

(b)

Figure 2. Structure of the EWD. (a) Pixel structure of the EWD. (b) Three-dimensional structure of an EWD panel.

In order to display different gray scales in EWDs, it is necessary to apply a voltage sequence for driving the pixel, the voltage sequence is called as driving waveform [14]. The driving waveform is designed as PWM (pulse width modulation) or AM (amplitude modulation) for displaying different gray scales accurately in EWDs [15]. The driving waveform affects the display effect of the EWD seriously. Figure 3 shows the conventional driving waveforms [9,10]. 


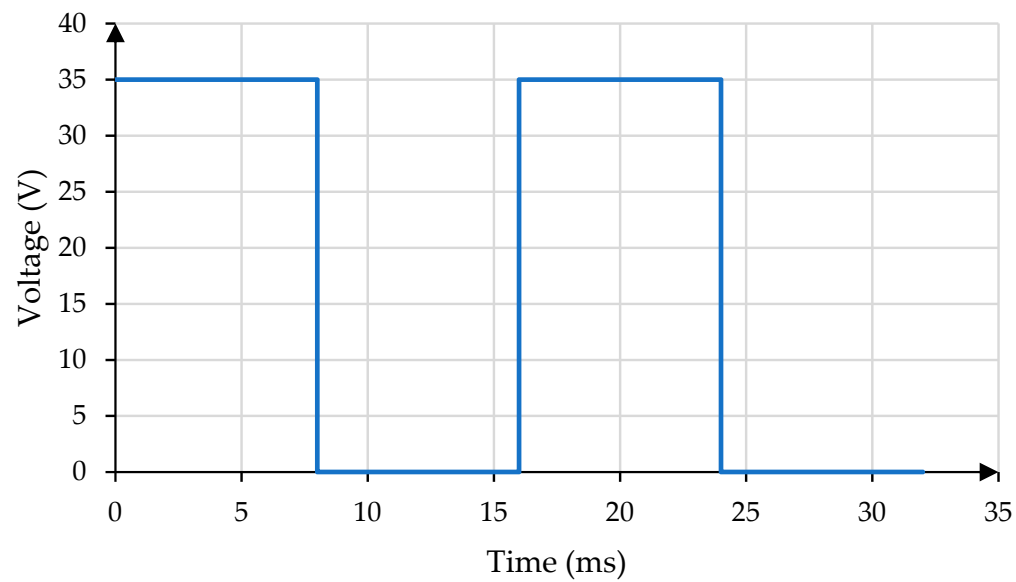

(a)

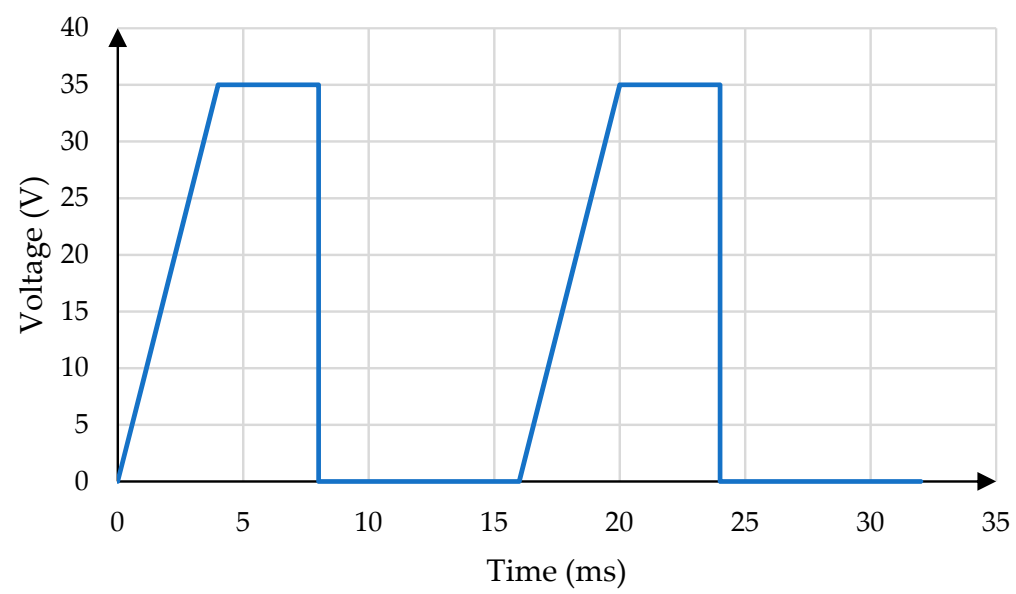

(b)

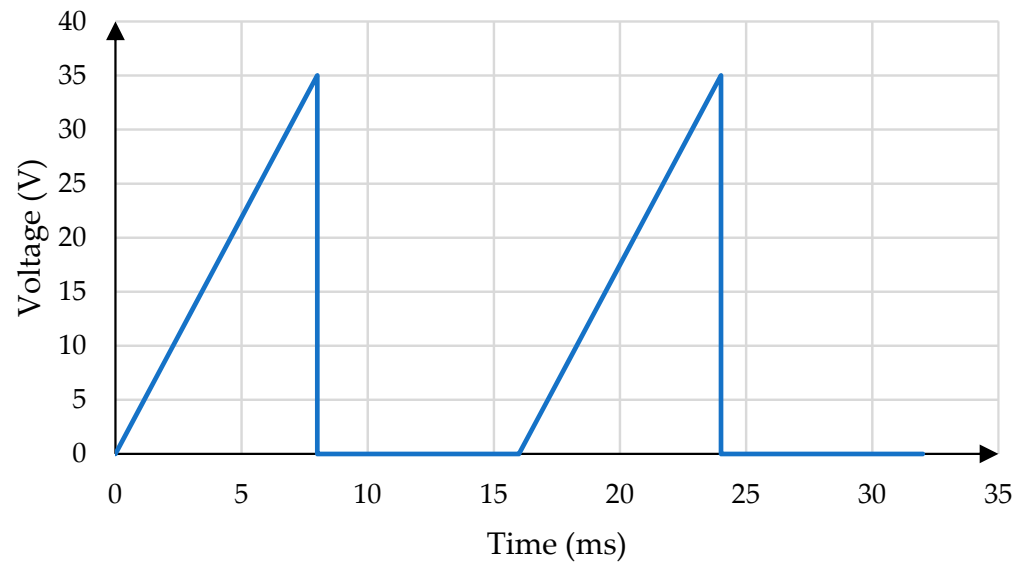

(c)

Figure 3. Conventional driving waveforms. (a) Square Wave. (b) Trapezoidal Wave. (c) Sawtooth Wave. 


\section{Driving Waveform Design for Low Power Consumption}

\subsection{Power Consumption Analysis}

As a capacitive device, the equivalent circuit model of an EWD pixel can be defined as a R-C (Resistance-Capacitance) circuit, as shown in Figure 4 [16]. In Figure $4 a, C_{p}$ represents the capacitance of the pixel unit, $R_{p}$ represents the resistance of the pixel unit, $R_{E}$ represents the external resistance, and the $\mathrm{E}$ is the driving voltage. Each pixel unit can be represented by a resistor connected in parallel with a capacitor. All pixel units are connected in series or parallel to form a whole. And then, the whole units are connected in series with an external resistance. This is the complete equivalent circuit model of the EWD. Figure $4 \mathrm{~b}$ is the simplified equivalent circuit model of an EWD pixel. $C_{S}$ represents the capacitance of an EWD pixel, $R_{S}$ represents the resistance of an EWD pixel.

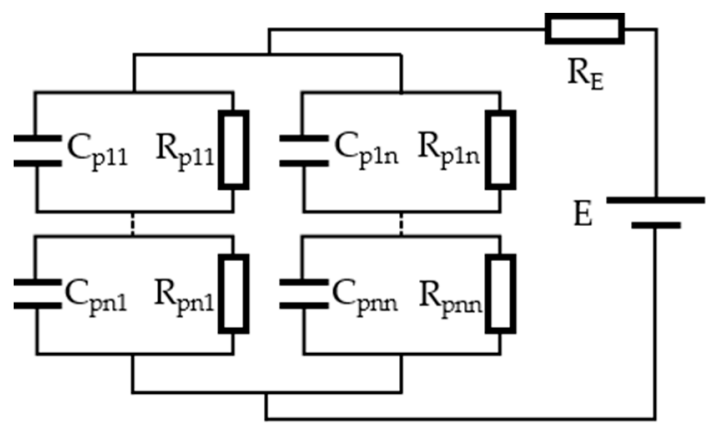

(a)

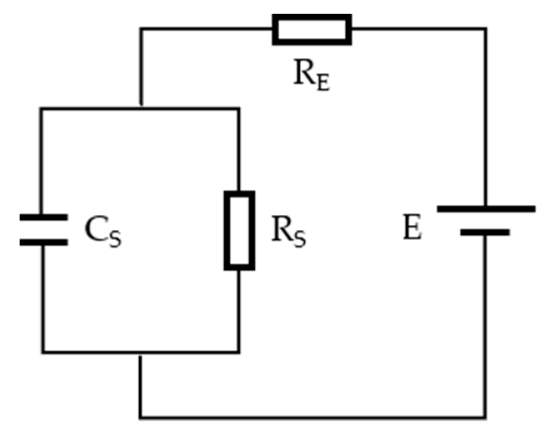

(b)

Figure 4. The equivalent circuit models of an EWD pixel. (a) Complete equivalent circuit model. (b) Simplified equivalent circuit model.

So, the power consumption of the simplified equivalent circuit can be shown as Equation (2).

$$
P=\frac{W}{T}=\frac{\frac{U^{2}}{R} t}{T}=\frac{U^{2} t}{T R}
$$

In Equation (2), $P$ represents the power consumption of the EWD, $W$ represents the electric energy consumed in a unit time $T, U$ represents the driving voltage, $t$ represents the effective driving time in a unit time $T$, and $R$ represents the impedance of the EWD. Because of the following theoretical analysis and experimental verification, the impedance of the EWD used in this paper is basically unchanged at the frequency of $60 \mathrm{~Hz}$.

According to the circuit model, an EWD pixel is an R-C series parallel circuit, as shown in Figure 5. There are two break frequencies $f 1$ and $f 2$ in R-C series parallel circuit [17,18], as shown in Equations (3) and (4).

$$
\begin{gathered}
f 1=\frac{1}{2 \pi R_{2} C_{E}} \\
f 2=\frac{1}{2 \pi C_{E}\left(\frac{R_{1} R_{2}}{R_{1}+R_{2}}\right)}
\end{gathered}
$$

In the Equations (3) and (4), $f 1$ and $f 2$ are the break frequencies, $C_{E}$ is the capacitance, $R_{1}$ and $R_{2}$ are series resistance and parallel resistance respectively.

When the frequency is lower than $f 1, C_{E}$ is equivalent to an open circuit, and the total impedance of the circuit is $R_{1}+R_{2}$. When the frequency is higher than $f 2, C_{E}$ is equivalent as a short circuit, and the total impedance of the circuit is $R_{1}$. When the frequency is higher than $f 1$ and lower than $f 2$, the total impedance of the circuit can be changed between $R_{1}+R_{2}$ and $R_{1}$. 


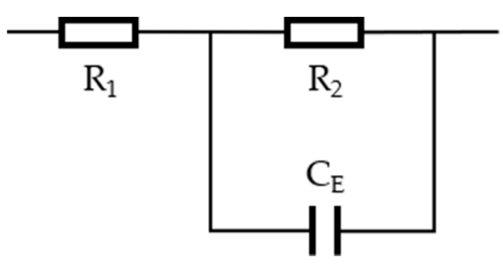

(a)

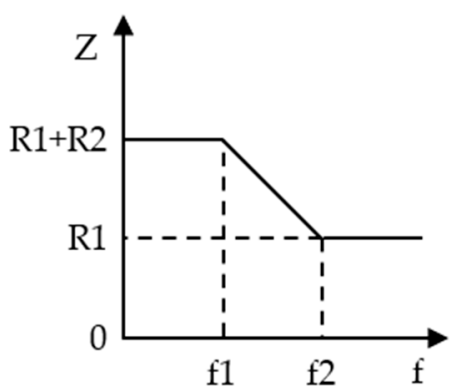

(b)

Figure 5. The impedance-frequency characteristics of the R-C series-parallel circuit. series-parallel circuit. (b) The relationship between the impedance and the break frequency.

(a) $\mathrm{R}-\mathrm{C}$

It can be seen from the Figure $5 b$ that the impedance remains unchanged at a fixed frequency. The impedance of the EWD used in this paper is $170.43 \mathrm{k} \Omega$ measured by the TH2828 Precision LCR Meter (Tonghui, Changzhou, China) at the frequency of $60 \mathrm{~Hz}$.

According to the above analysis, the driving power of the EWD is mainly determined by the driving voltage $U$ and the effective driving time $t$, which can be expressed as the Equation (5).

$$
P=\frac{1}{T R} \int_{0}^{T} U^{2}(t) d t
$$

where $U(t)$ is the voltage of the driving waveform at time $t$.

\subsection{Power Consumption Analysis of Conventional Driving Waveforms}

For conventional driving waveforms, such as square wave and trapezoidal wave, their expressions and power consumption expressions can be derived.

Square wave is shown in Equation (6). $C_{1}$ is the voltage constant from 0 to $\frac{1}{2} T$, and $C_{2}$ is the voltage constant from $\frac{1}{2} T$ to $T$.

$$
U(t)= \begin{cases}C_{1}, & 0 \leq t<\frac{1}{2} T \\ C_{2}, & \frac{1}{2} T \leq t<T\end{cases}
$$

Then, the power consumption expression of Square Wave can be obtained, as shown in Equation (7).

$$
P=\frac{1}{T R} \int_{0}^{\frac{1}{2} T} C_{1}^{2} d t+\frac{1}{T R} \int_{\frac{1}{2} T}^{T} C_{2}^{2} d t
$$

Trapezoidal wave is shown in Equation (8). $k$ is the slope between 0 and $\frac{1}{4} T, C_{1}$ is the voltage constant from $\frac{1}{4} T$ to $\frac{1}{2} T$, and $C_{2}$ is the voltage constant from $\frac{1}{2} T$ to $T$.

$$
U(t)=\left\{\begin{array}{lc}
k t, \quad 0 \leq t<\frac{1}{4} T \\
C_{1}, \quad \frac{1}{4} T \leq t<\frac{1}{2} T \\
C_{2}, \quad \frac{1}{2} T \leq t<T
\end{array}\right.
$$

Then, the power consumption expression of trapezoidal wave can be obtained, as shown in Equation (9).

$$
P=\frac{1}{T R} \int_{0}^{\frac{1}{4} T}(k t)^{2} d t+\frac{1}{T R} \int_{\frac{1}{4} T}^{\frac{1}{2} T} C_{1}^{2} d t+\frac{1}{T R} \int_{\frac{1}{2} T}^{T} C_{2}^{2} d t
$$


Sawtooth wave is shown in Equation (10). $k$ is the slope between 0 and $\frac{1}{2} T, C_{1}$ is the voltage constant from $\frac{1}{2} T$ to $T$.

$$
U(t)= \begin{cases}k t, & 0 \leq t<\frac{1}{2} T \\ C_{1}, & \frac{1}{2} T \leq t<T\end{cases}
$$

Then, the power consumption expression of Sawtooth Wave can be obtained, as shown in Equation (11).

$$
P=\frac{1}{T R} \int_{0}^{\frac{1}{2} T}(k t)^{2} d t+\frac{1}{T R} \int_{\frac{1}{2} T}^{T} C_{1}^{2} d t
$$

\subsection{Driving Waveform Design}

The driving waveform for low power consumption is a sequence of voltages. In order to design a perfect driving waveform, not only the frequency, amplitude and duty cycle of the waveform should be considered, but also the inherent defects of the EWD should be avoided by appropriate optimization and adjustment. In the proposed driving waveform, the frequency is set as $60 \mathrm{~Hz}$ [19], the voltage can be changed between 0 and $35 \mathrm{~V}$, and the duty is set as $50 \%$. Based on the square wave, the proposed driving waveform is shown in Figure 6a, which consists of three stages.

The first stage is a linear rising process. The driving voltage is changed from $10 \mathrm{~V}$ to $35 \mathrm{~V}$ in $5 \mathrm{~ms}$ with a linear gradient. The $10 \mathrm{~V}$ is the threshold voltage for driving the oil. The oil is in a balance state of natural spreading when no voltage is applied. In order to break this balance state, the threshold voltage needs to be applied. As shown in Figure 6b, the static balance can be broken when the driving voltage is improved to $10 \mathrm{~V}$.

In addition, the rising gradient from $10 \mathrm{~V}$ to $35 \mathrm{~V}$ is added for reducing the effect of oil rupture on the aperture ratio [10]. At the same time, it can reduce the duration of the driving cycle. The aperture ratio of different rising gradients is shown in Figure 6c. The rising duration is designed as $5 \mathrm{~ms}$ for reducing power consumption.

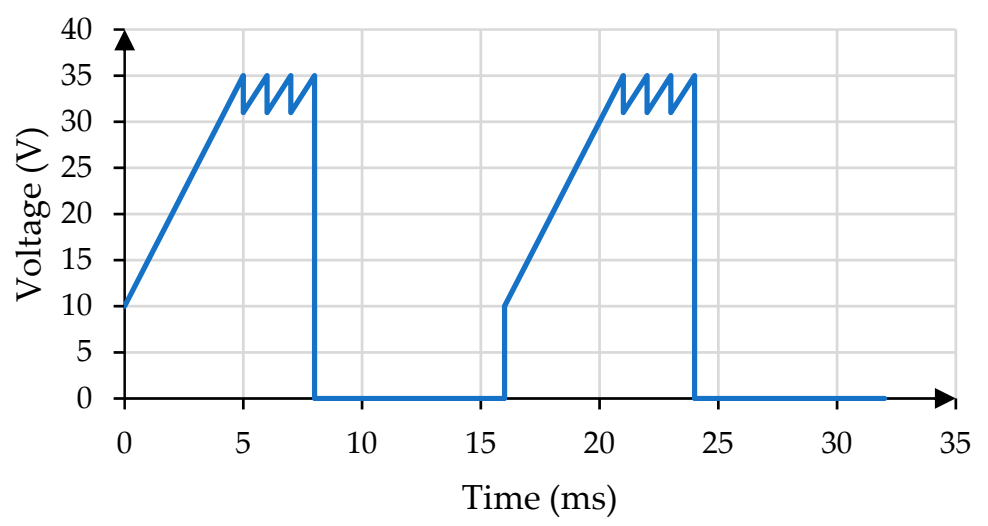

(a)

Figure 6. Cont. 


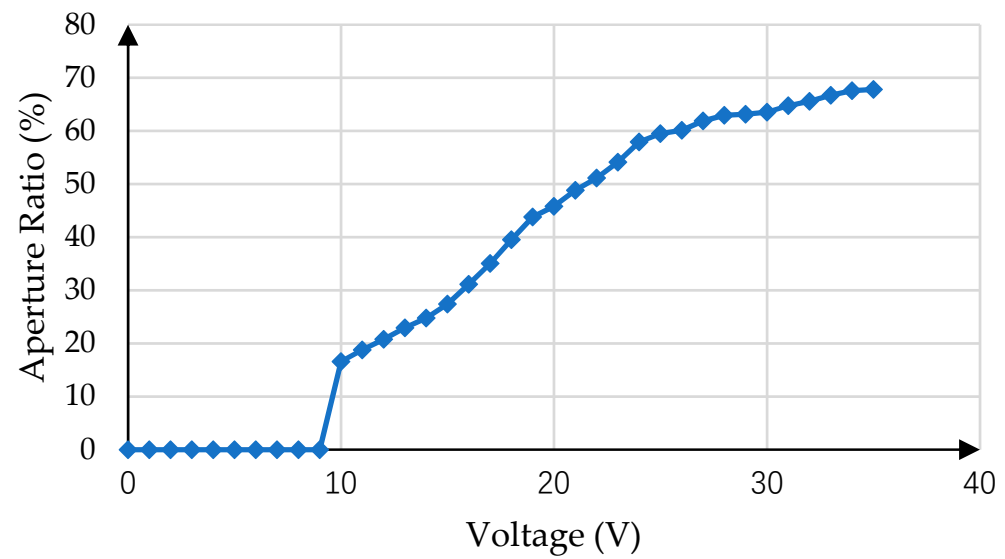

(b)

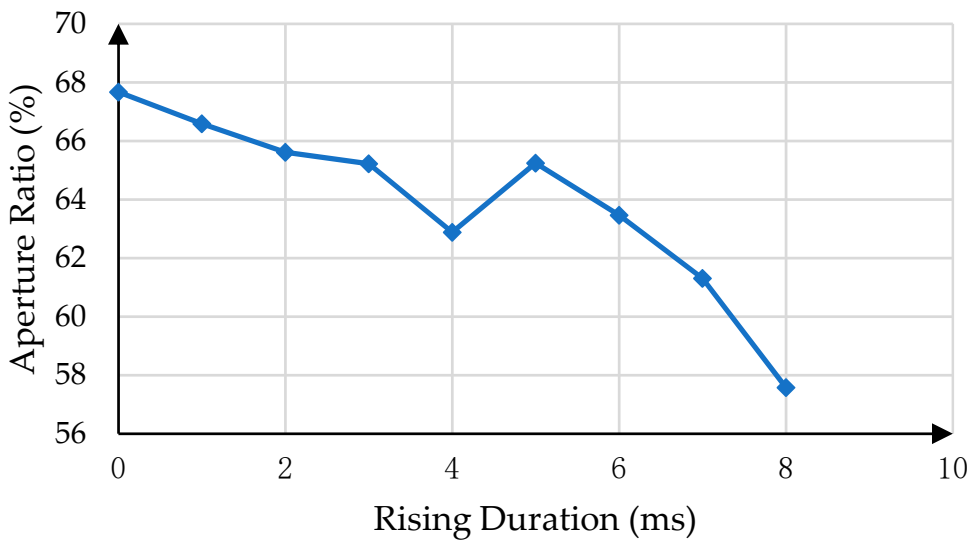

(c)

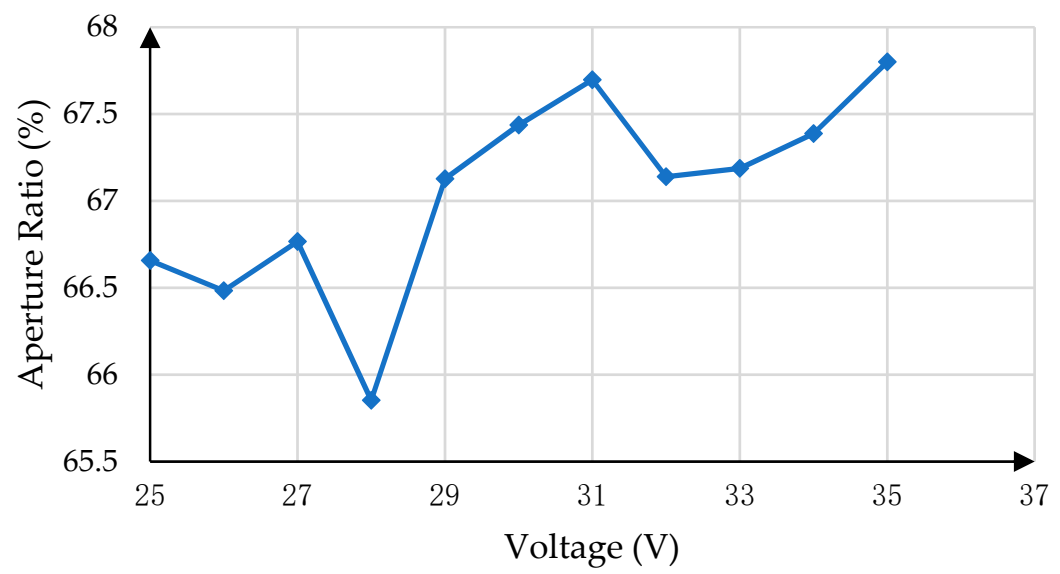

(d)

Figure 6. Design of the proposed driving waveform. (a) The proposed driving waveform for low power consumption. (b) The relationship between the voltage and the aperture ratio. (c) The relationship between the rising duration and the aperture ratio. (d) The relationship between minimum driving voltage and Aperture Ratio in the sawtooth wave. 
The second stage is a periodic change process. The driving voltage is changed between $31 \mathrm{~V}$ and $35 \mathrm{~V}$ in $3 \mathrm{~ms}$, which is a sawtooth wave. The sawtooth wave can reduce the driving voltage for saving the power consumption and inhibit the oil backflow to a certain extent. The oil will backflow due to charge trapping when the same voltage is applied continuously [8]. The $31 \mathrm{~V}$ is the minimum driving voltage of the sawtooth wave. Although the lower driving voltage can reduce more power consumption, the lower driving voltage can reduce the aperture ratio. In order to obtain higher aperture ratio and lower power consumption, $31 \mathrm{~V}$ is selected as the minimum driving voltage for the sawtooth wave. The relationship between minimum driving voltage of the sawtooth wave and aperture ratio is shown in Figure $6 \mathrm{~d}$, and a higher aperture ratio can be obtained at $31 \mathrm{~V}$. In order to achieve a higher aperture ratio, a higher voltage is needed, but the oil will backflow when a constant voltage is applied to the EWD. And the sawtooth wave can inhibit the oil backflow to a certain extend. So, the aperture ratio will fluctuate locally under the driving of constant voltage and sawtooth wave. Further, for different EWDs, the minimum driving voltages of the sawtooth wave are different, which can be obtained by the same experimental method.

The third stage is a resetting process. The driving voltage is held at $0 \mathrm{~V}$ for $8 \mathrm{~ms}$ to eliminate the influence of charge leakage [9].

According to the proposed driving waveform, its expression can be expressed as Equation (12). $k_{1}$ is the slop, $b_{1}$ is the intercept between 0 and $\frac{5}{16} T, k_{2}$ is the slop, $b_{2}$ is the intercept between $\frac{5}{16} T$ and $\frac{1}{2} T$, $C$ represents the voltage constant from $\frac{1}{2} T$ to $T$.

$$
U(t)= \begin{cases}k_{1} t+b_{1}, & 0 \leq t<\frac{5}{16} T \\ k_{2}(t-a)+b_{2}, & \frac{5}{16} T \leq t<\frac{1}{2} T \\ C, & \frac{1}{2} T \leq t<T\end{cases}
$$

Then, the power consumption expression of the proposed driving waveform can be expressed as Equation (13).

$$
P=\frac{1}{R} \int_{0}^{\frac{5}{16} T}\left(k_{1} t+b_{1}\right)^{2} d t+\frac{1}{R} \int_{\frac{5}{16} T}^{\frac{1}{2} T}\left[k_{2}(t-a)+b_{2}\right]^{2} d t+\frac{1}{R} \int_{\frac{1}{2} T}^{T} C^{2} d t
$$

\section{Experimental Results and Discussion}

For the sake of evaluating the performance of the proposed driving waveform, an experimental platform is developed to measure the aperture ratio and the power consumption of EWDs, as shown in Figure 7. This experimental platform includes a driving system, a measuring system, and an EWD panel. The driving system, which is used to input the driving waveform for driving the EWD, consists of a computer, a function generator, and a high-voltage amplifier. The testing system is used to measure and record test results, including a microscope, a high-speed camera, a power meter, and a computer, and the EWD is the measured object. Its parameters are shown in the Table 1.

The experimental process can be divided into two steps. Firstly, the EWD is driven by the driving system. The driving waveform is edited by a computer with ArbexPress software, and it is sent to function generator by serial communication, and then, the driving voltage in the driving waveform can be output when it is amplified by the high-voltage amplifier for driving the EWD. Secondly, the experimental data is measured by the testing system. The high-speed camera captures the display state of the EWD in real time by the microscope and transmits testing data to the computer for calculating the aperture ratio. Meanwhile, the power meter measures and records the power consumption of the EWD panel in real time. During the experiment, the temperature is kept constant to avoid the influence of the external environment.

The aperture ratio and power consumption of the EWD with different driving waveforms are measured by the above-mentioned experimental platform. The measurement results are shown in Figure 8. 


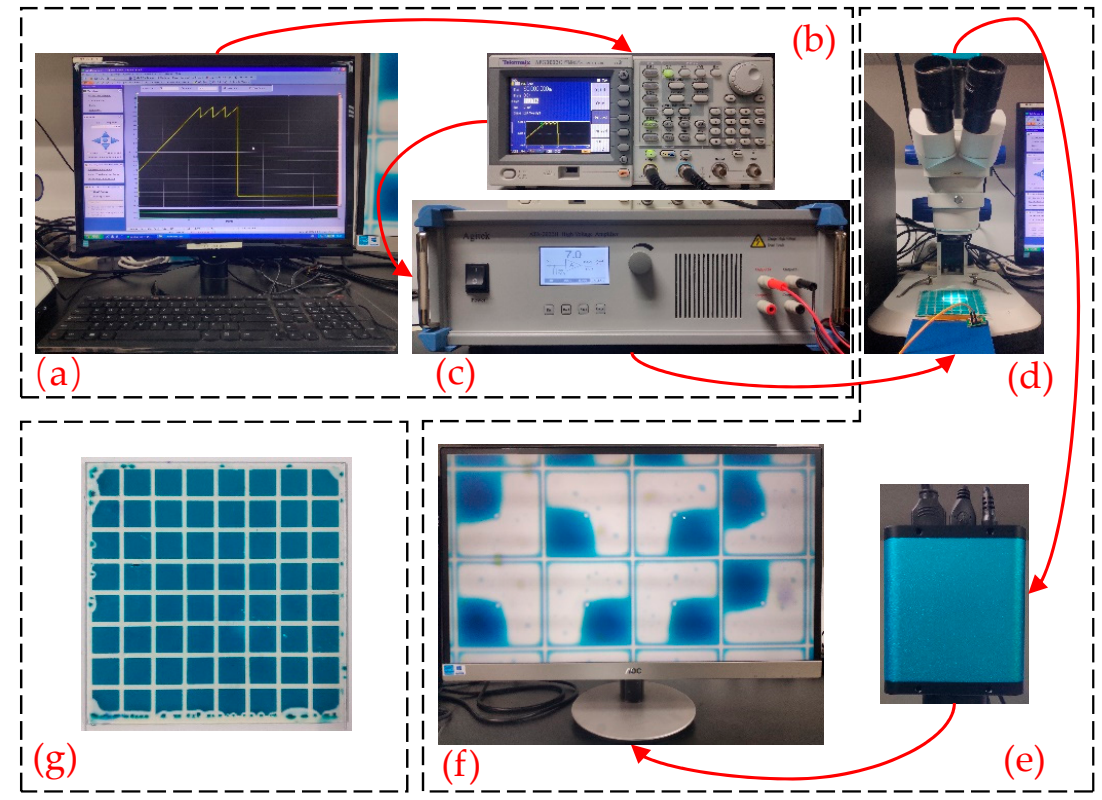

Figure 7. Experimental platform of the driving waveform for EWDs. (a) A computer for driving system. (b) Arbitrary function generator ATA-2022H (Agitek, Xi'an, China). (c) AFG-3052C (Agitek, Xi'an, China) high voltage amplifier. (d) Microscope. (e) High speed camera. (f) PC for measuring system. (g) An EWD panel.

Table 1. Parameters of the electrowetting display (EWD) panel.

\begin{tabular}{cccccccc}
\hline Panel Size & Oil Color & Resolution & Pixel Size & $\begin{array}{c}\text { Pixel } \\
\text { Wall Size }\end{array}$ & $\begin{array}{c}\text { Pixel } \\
\text { Wall Height }\end{array}$ & $\begin{array}{c}\text { Hydrophobic } \\
\text { Layer Thickness }\end{array}$ & $\begin{array}{c}\text { Driving } \\
\text { Voltage }\end{array}$ \\
\hline $10 \times 10 \mathrm{~cm}$ & Cyan & $200 \times 200$ & $150 \times 150 \mathrm{um}$ & $15 \times 15 \mathrm{um}$ & $5.6 \mathrm{um}$ & $1 \mathrm{um}$ & $0-35 \mathrm{~V}$ \\
\hline
\end{tabular}
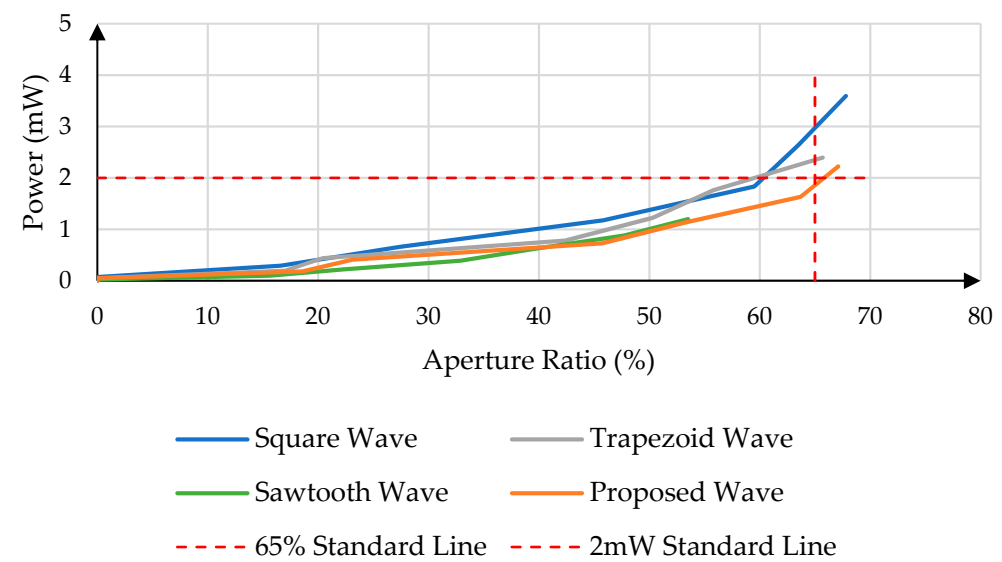

Figure 8. The relationship between Aperture Ratio and Power Consumption.

As can be seen from Figure 8, with the same aperture ratio, the power consumption of the proposed driving waveform is lower than that of the conventional driving waveforms for the EWD. As shown in Figure 9, the aperture ratio of the proposed driving waveform is larger than that of conventional driving waveforms when the power consumption is $2 \mathrm{~mW}$.

More experiments are designed to further illustrate the characteristics of the proposed driving waveform. As shown in Figure 10a, with the increase of the aperture ratio, the influence of the voltage on it decreases gradually. And as can be seen from the Figure 10b, the driving frequency also have an effect on the aperture ratio. The aperture ratio increases with the increase of driving frequency, but there will be local fluctuation. Figure 10c shows the relationship between aperture ratio and power. 


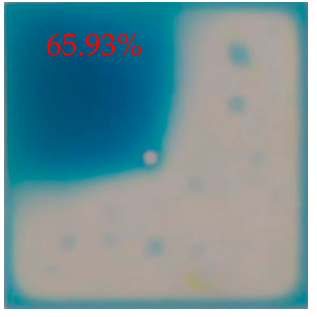

(a)

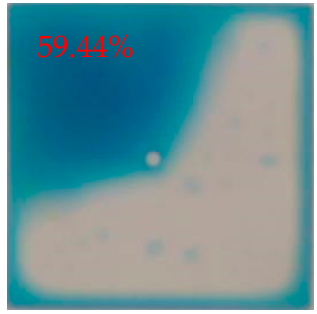

(b)

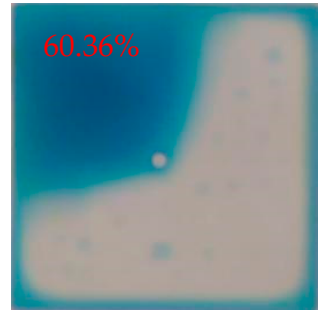

(c)

Figure 9. Aperture ratio with different driving waveforms under the same power consumption. (a) The proposed driving waveform. (b) Trapezoid driving waveform. (c) Square driving waveform.

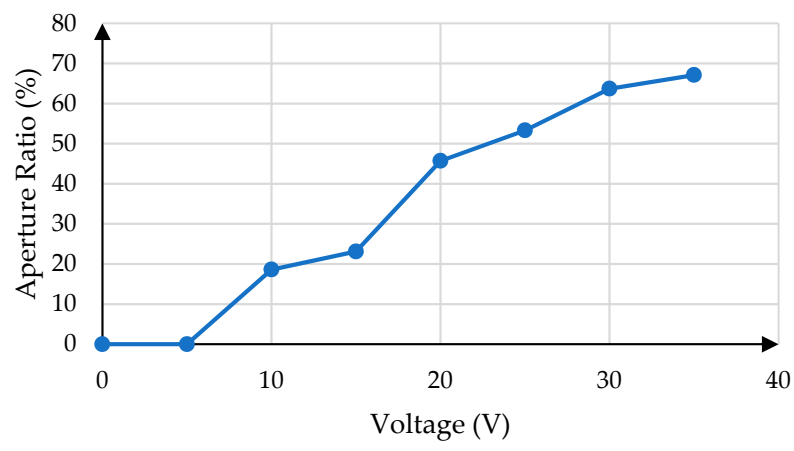

(a)

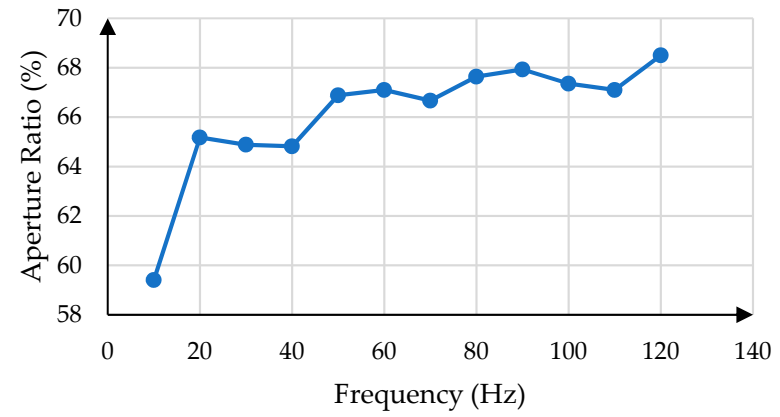

(b)

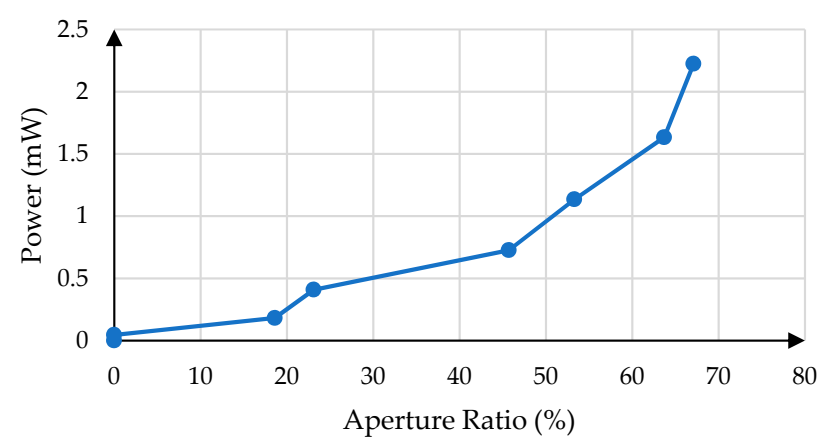

(c)

Figure 10. Characteristics of the proposed driving waveform. (a) The relationship between voltage and aperture ratio. (b) The relationship between frequency and aperture ratio. (c) The relationship between aperture ratio and power. 
Similarly, the structural parameters of the EWD can affect its power consumption and aperture ratio. However, the shape of the proposed driving waveform cannot be affected, nor will it affect the result in that the proposed driving waveform has lower power consumption than the conventional square wave under the same aperture ratio.

\section{Conclusions}

In this paper, the power consumption of the driving waveform for EWDs is analyzed, and the relationship between the driving waveform and the power consumption is obtained. Then, a new driving waveform with a rising gradient and a sawtooth wave is proposed. The rising gradient can prohibit the oil breakup effectively. The section of sawtooth wave can inhibit the backflow of the oil. Experimental results show that the proposed driving waveform has a lower power consumption and higher aperture ratio than conventional waveforms. When the aperture ratio is $65 \%$, the power consumption of the proposed driving waveform is about $38.13 \%$ lower than that of the conventionally used square wave. In addition, the proposed driving waveform can provide a new way for the optimization of the EWD driving system.

Author Contributions: W.L. and Z.Y. designed this project. W.L. and L.W. carried out most of the experiments and data analysis. T.Z., S.L., L.L. and W.H. performed part of the experiments and for help discussion during manuscript preparation. L.W. and Z.Y. contributed to the data analysis and correction. G.Z. gave suggestions on the project management and conducted helpful discussion on the experimental results. All authors have read and agree to the published version of the manuscript.

Funding: This research was funded by National Key R\&D Program of China (No. 2016YFB0401502), the Key Research Platforms and Research Projects in Universities and Colleges of Guangdong Provincial Department of Education (No. 2018KQNCX334), Zhongshan Innovative Research Team Program (No. 180809162197886), Zhongshan Institute high-level talent scientific research startup fund project (No. 416YKQ04), National Key R\&D Program of China (No. 2018YFB0407100-02, No. 2018YFB1801302), Key-Area Research and Development Program of GuangDong Province (2019B010924005), Program for Chang Jiang Scholars and Innovative Research Teams in Universities (No. IRT_17R40), Science and Technology Program of Guangzhou (No. 2019050001), Guangdong Provincial Key Laboratory of Optical Information Materials and Technology (No. 2017B030301007), MOE International Laboratory for Optical Information Technologies and the 111 Project.

Conflicts of Interest: The authors declare no conflict of interest.

\section{References}

1. Hayes, R.A.; Feenstra, B.J. Video-speed electronic paper based on electrowetting. Nature 2003, 425, 383. [CrossRef] [PubMed]

2. Beni, G.; Hackwood, S. Electro-wetting displays. Appl. Phys. Lett. 1981, 38, 207-209. [CrossRef]

3. You, H.; Steckl, A.J. Three-color electrowetting display device for electronic paper. Appl. Phys. Lett. 2010, 97, 023514. [CrossRef]

4. Seyrat, E.; Hayes, R.A. Amorphous fluoropolymers as insulators for reversible low-voltage electrowetting. J. Appl. Phys. 2001, 90, 1383-1386. [CrossRef]

5. Roques-Carmes, T.; Hayes, R.A.; Feenstra, B.J.; Schlangen, L.J. Liquid behavior inside a reflective display pixel based on electrowetting. J. Appl. Phys. 2004, 95, 4389-4396. [CrossRef]

6. Roques-Carmes, T.; Palmier, S.; Hayes, R.A.; Schlangen, L.J. The effect of the oil/water interfacial tension on electrowetting driven fluid motion. Colloids Surf. A Phys. Eng. Asp. 2005, 267, 56-63. [CrossRef]

7. Sureshkumar, P.; Kim, M.; Song, E.G.; Lim, Y.J.; Lee, S.H. Effect of surface roughness on the fabrication of electrowetting display cells and its electro-optic switching behavior. Surf. Rev. Lett. 2009, 16, 23-28. [CrossRef]

8. Liang, C.C.; Chen, Y.C.; Chiu, Y.H.; Chen, H.Y.; Cheng, W.Y.; Lee, W.Y. 27.3: A Decoupling Driving Scheme for Low Voltage Stress in Driving a Large-Area High-Resolution Electrowetting Display. In SID Symposium Digest of Technical Papers; Blackwell Publishing Ltd.: Oxford, UK, 2009; Volume 40, pp. 375-378.

9. Yi, Z.; Shui, L.; Wang, L.; Jin, M.; Hayes, R.A.; Zhou, G. A novel driver for active matrix electrowetting displays. Displays 2015, 37, 86-93. [CrossRef]

10. Zhang, X.M.; Bai, P.F.; Hayes, R.A.; Shui, L.L.; Jin, M.L.; Tang, B.; Zhou, G.F. Novel driving methods for manipulating oil motion in electrofluidic display pixels. J. Disp. Technol. 2015, 12, 200-205. [CrossRef] 
11. Luo, Z.J.; Zhang, W.N.; Liu, L.W.; Xie, S.; Zhou, G. Portable multi-gray scale video playing scheme for high-performance electrowetting displays. J. Soc. Inf. Disp. 2016, 24, 345-354. [CrossRef]

12. Feenstra, J. Video-Speed Electrowetting Display Technology. In Handbook of Visual Display Technology; Springer International Publishing: New York, NY, USA, 2014; pp. 1-13. [CrossRef]

13. Dou, Y.; Tang, B.; Groenewold, J.; Li, F.; Yue, Q.; Zhou, R.; Li, H.; Shui, L.; Henzen, A.; Zhou, G. Oil motion control by an extra pinning structure in electro-fluidic display. Sensors 2018, 18, 1114. [CrossRef] [PubMed]

14. Shen, S.; Gong, Y.; Jin, M.; Yan, Z.; Xu, C.; Yi, Z.; Zhou, G.; Shui, L. Improving electrophoretic particle motion control in electrophoretic displays by eliminating the fringing effect via driving waveform design. Micromachines 2018, 9, 143. [CrossRef] [PubMed]

15. Chiu, Y.H.; Liang, C.C.; Chen, Y.C.; Lee, W.Y.; Chen, H.Y.; Wu, S.H. Accurate-gray-level and quick-response driving methods for high-performance electrowetting displays. J. Soc. Inf. Disp. 2011, 19, 741-748. [CrossRef]

16. Zhao, Q.; Tang, B.; Dong, B.; Li, H.; Zhou, R.; Guo, Y.; Dou, Y.; Deng, Y.; Groenewold, J.; Henzen, A.V.; et al. Electrowetting on dielectric: Experimental and model study of oil conductivity on rupture voltage. J. Phys. D Appl. Phys. 2018, 51, 195102. [CrossRef]

17. Chen, C.H.; Tsai, S.L.; Chen, M.K.; Jang, L.S. Effects of gap height, applied frequency, and fluid conductivity on minimum actuation voltage of electrowetting-on-dielectric and liquid dielectrophoresis. Sens. Actuators B Chem. 2011, 159, 321-327. [CrossRef]

18. Kumar, A.; Pluntke, M.; Cross, B.; Baret, J.C.; Mugele, F. Finite conductivity effects and apparent contact angle saturation in AC electrowetting. MRS Online Proc. Libr. Arch. 2005. [CrossRef]

19. Van Dijk, R.; Feenstra, B.J.; Hayes, R.A.; Camps, I.G.J.; Boom, R.G.H.; Wagemans, M.M.H.; Giraldo, A.; Heijden, B.V.; Los, R.; Feil, H. 68.3: Gray scales for video applications on electrowetting displays. In SID Symposium Digest of Technical Papers; Blackwell Publishing Ltd.: Oxford, UK, 2006; Volume 37, pp. 1926-1929.

(C) 2020 by the authors. Licensee MDPI, Basel, Switzerland. This article is an open access article distributed under the terms and conditions of the Creative Commons Attribution (CC BY) license (http://creativecommons.org/licenses/by/4.0/). 